\title{
Aura sin migraña o síndrome de Alicia en el País de las Maravillas. Caso clínico pediátrico
}

\author{
Aura without migraine or Alice in Wonderland syndrome. \\ Pediatric clinical case
}

\author{
Dr. Daniel Gómez-Sáncheza , Dr. Gonzalo Ros-Cerverab, Dra. Julia Pérez-Verdúc, \\ Dra. Francisca Pelechano-Ribes ${ }^{b}$, Dr. Nelson Orta-Sibúd y Dr. José M. Sequí Canete
}

\begin{abstract}
RESUMEN
La migraña con aura en pacientes pediátricos es reportada con frecuencia; el aura típica sin migraña, en raras ocasiones, y el aura persistente asociada al denominado síndrome de Alicia en el País de las Maravillas, excepcionalmente.

El objetivo de este reporte es presentar un caso clínico correspondiente a la última variedad mencionada. Se trata de una paciente de 6 años de edad, quien tuvo, al menos, dos episodios con estas características antes de presentar un cuadro clínico completo de aura y, consecutivamente, cefalea con más frecuencia. Los estudios complementarios practicados de manera sistemática a la paciente fueron normales, con mejoría clínica con el tratamiento convencional.

Se debe sospechar el síndrome en todo paciente que se presente con las características descritas a fin de aproximarse al diagnóstico de aura sin migraña, descartar patologías orgánicas o de otra naturaleza e instaurar la terapéutica adecuada.

Palabras clave: migraña con aura, niño, sindrome de Alicia en el País de las Maravillas.
\end{abstract}

\begin{abstract}
Migraine with aura in children is often reported, typical aura without headache is reported sometimes, but persistent aura and Alice in Wonderland syndrome is exceptionally reported. We present a case corresponding to the last one mentioned in a 6-year-old patient who had at least two episodes with the typical characteristics of aura without migraine before developing more frequently the complete clinical picture of aura and subsequently headache. The complementary studies systematically done to the patient were normal and she improved with conventional therapy.
\end{abstract}

a. Unidad de Cefaleas.

b. Unidad de Neuropediatría.

c. Servicio de Pediatría General.

d. Profesor titular visitante.

e. Sección de Pediatría.

Hospital "Francisco de Borja", Gandía, Valencia, España.

Correspondencia:

Dr. Daniel Gómez-Sánchez: dgomezsan@gmail.com

Financiamiento: Ninguno.

Conflicto de intereses: Ninguno que declarar.

Recibido: 15-11-2017

Aceptado: 11-8-2018
It is important to emphasize that the syndrome should be suspected in any patient who presents the described manifestations in order to approach to the diagnosis of aura without migraine, to discard either organic pathology or another cause and to indicate adequate therapeutic measures.

Keywords: migraine with aura, child, Alice in Wonderlandsyndrome.

http: / / dx.doi.org/10.5546/aap.2019.e34

Cómo citar: Gómez-Sánchez D, Ros-Cervera G, Pérez-Verdú J, Pelechano-Ribes F, et al. Aura sin migraña o síndrome de Alicia en el País de las Maravillas. Caso clínico pediátrico. Arch Argent Pediatr 2019;117(1):e34-e36.

\section{INTRODUCCIÓN}

La migraña es una cefalea primaria, sin causa aparente, ${ }^{1}$ que afecta alrededor del 5-10\% de los niños..$^{2-5}$ De acuerdo con la Clasificación Internacional de Cefaleas (International Classification of Headache Disorders; ICHD III, por sus siglas en inglés), ${ }^{6}$ la migraña se clasifica, a grandes rasgos, en migraña con aura o sin aura, pero esta entidad no siempre se manifiesta de forma clara y puede aparecer, en muy pocos casos, en forma de aura aislada como único síntoma y presentarse como el denominado síndrome de Alicia en el País de las Maravillas, ${ }^{1}$ lo cual dificulta su diagnóstico, al que se llega por exclusión tras descartar otras causas de organicidad.

El objetivo es presentar y discutir un caso clínico y aportar detalles del aura sin migraña o síndrome de Alicia en el País de las Maravillas, así como la dinámica necesaria para una aproximación diagnóstica.

\section{CASO CLÍNICO}

Paciente femenina de 6 años de edad, controlada en consultas externas de Oftalmología por hipermetropía y astigmatismo, quien acudió a Urgencias de Pediatría por presentar visión en blanco y negro, de una hora de evolución, sin otra clínica asociada. Los padres negaron la presencia de fiebre, sin clínica catarral ni gastrointestinal, y 
la exploración física y neurológica fue normal en todo momento.

La niña refirió que veía solo en blanco y negro, y que no era capaz de apreciar el color de las cosas, pero no presentaba otras alteraciones ni de la forma ni del tamaño de los objetos. Aunque expresó miedo, los padres no percibieron sensación de terror y, por la edad de la paciente, no se precisó si presentaba distorsión de la imagen corporal. Los padres comentaron que esto ya le había ocurrido en otra ocasión, pero, dado que el cuadro había desaparecido en pocas horas, le habían restado importancia.

Este nuevo episodio tuvo una duración de 5 horas, con recuperación completa posterior y sin ningún otro síntoma asociado. Específicamente, no se acompañó de cefalea ni antes de la alteración visual, ni durante ella, ni en las horas siguientes.

Además, el rendimiento escolar de la paciente era bueno, no tenía antecedentes de convulsiones ni tomaba ninguna medicación de base. Como antecedentes personales de interés, se destacaba que, a los 2 años, había tenido un traumatismo craneoencefálico leve, que no había requerido internación, y que, en ocasiones, presentaba dolor de cabeza de localización en la región temporal, que se resolvía de forma espontánea, durmiendo o con la administración de una dosis terapéutica de paracetamol. Dicho dolor aparecía antes de los exámenes escolares, no ocurría durante las vacaciones ni interfería en su actividad habitual y no presentaba signos de alerta que sugirieran una patología orgánica cerebral.

Como antecedente familiar de interés, referían que la madre era migrañosa.

Las pruebas complementarias realizadas de fondo de ojo, electroencefalograma, tomografía axial computarizada de cráneo, resonancia magnética cerebral fueron normales, y la serología para citomegalovirus, virus de Epstein-Barr y varicela fue negativa.

A los 2 meses de este cuadro, mientras se encontraba en seguimiento y en estudio en la consulta externa, presentó, de nuevo, un episodio de visión en blanco y negro, seguido, a los 30 minutos, de cefalea de localización frontal, pulsátil, de unas 6 horas de duración, acompañada de fotofobia, fonofobia y náuseas, que se resolvió tras el reposo en decúbito en un ambiente tranquilo y con la administración de una dosis terapéutica de ibuprofeno por vía oral.

A partir de este episodio, la niña ha presentado cefalea de características migrañosas en otras ocasiones (1-2 episodios al mes), no siempre acompañada de alteración visual, de predominio vespertino, con normalidad clínica, neurológica y oftalmológica entre los episodios.

\section{DISCUSIÓN}

La prevalencia de aura en la migraña en pediatría es variable y oscila entre el $10 \%$ y el 30 $\%, 7$ según los criterios diagnósticos que se utilicen. La variedad de aura aislada o acefalálgica es una forma poco frecuente en la población adulta y excepcional en pediatría. ${ }^{6,7}$

Se define aura como la aparición de uno o más síntomas visuales, sensitivos o difásicos, de carácter reversible, que ocurren de forma gradual a lo largo de 5 a 20 minutos previos a la crisis de cefalea y desaparecen en menos de 60 minutos. $^{8}$

Entre los síntomas visuales, se pueden encontrar hallazgos positivos (líneas, manchas, luces centelleantes, etc.) y/o negativos (visión borrosa, escotomas, etc.), distorsiones del tamaño (micropsias, macropsias) y de la forma (metamorfopsias). Entre los sensitivos, puede haber síntomas positivos (parestesias) y/o negativos (hipoestesias). ${ }^{4-6}$

En general, en la bibliografía sobre aura migrañosa presentada como síndrome de Alicia en el País de las Maravillas sin cefalea asociada, la frecuencia cae al 0,57 \%.9

Este síndrome fue descrito preliminarmente como manifestación por Lippman en $1952^{10}$ y, luego, en 1955, por Todd, ${ }^{11}$ quien le dio el nombre de síndrome de Alicia en el País de las Maravillas en honor a Charles Dodgson, autor de esta novela escrita bajo el pseudónimo de Lewis Carroll. Dodgson sufría de migrañas, y, quizás, este síndrome lo habría inspirado a escribir esta novela y reflejar algunas alteraciones en la percepción de los personajes.

El síndrome se define como un cuadro complejo que se caracteriza por alteraciones de la percepción visual, como alteraciones de la forma de los objetos (metamorfopsia), del tamaño, del color, de la orientación espacial (teleopsia), de la imagen corporal (macro- y microsomatognosia), sensación de visión invertida, incapacidad para reconocer caras (prosopagnosia), pérdida de visión estereoscópica, sensación de tener dos cuerpos que pueden ir acompañados de episodios de desrealización, despersonalización y alteración de la percepción del tiempo. El paciente es consciente de la naturaleza irreal de su percepción. ${ }^{12}$

En lo referente a la etiología, ${ }^{13}$ clásicamente, se ha asociado a la migraña ${ }^{14,15}$ y a la epilepsia, 
aunque también a otras causas de lesión cerebral (aneurismas, tumores), infecciones virales, sobre todo, por virus de Epstein-Barr, aunque también por otros, como citomegalovirus, varicela zóster, coxsackie e influenza, fármacos (montelukast, topiramato, risperidona, clomifeno, antidepresivos y drogas, como ácido lisérgico, éxtasis y marihuana). ${ }^{12,13}$

En la etiología migrañosa, como en nuestro caso, por lo general, hay antecedentes familiares, principalmente, en la madre, y también personales. El síndrome suele preceder a la migraña y presentarse como aura, aunque no siempre seguido de cefalea. ${ }^{13-15}$

En la etiología epiléptica, se debe hacer un diagnóstico diferencial con crisis focales occipitales. Se diferencian de la migraña en que la epilepsia occipital dura apenas unos segundos y se manifiesta en forma de círculos y esferas y con alteración del color de los objetos. ${ }^{8}$

Ante un paciente con sospecha de síndrome de Alicia en el País de las Maravillas, se debe actuar como en cualquier proceso de diagnóstico médico, con la anamnesis, la exploración física y el estudio complementario en función de la sospecha clínica. ${ }^{14}$

En primer lugar, se debe indagar sobre lo que le ocurre al paciente y desde cuándo, ${ }^{13}$ investigar los síntomas asociados, si se acompaña de síntomas infecciosos sugestivos de cuadros fundamentalmente de naturaleza viral por los agentes ya mencionados y solicitar la serología pertinente. ${ }^{13-15}$

Se deben investigar datos sugestivos de alteraciones de la refracción o patología ocular a través de una interconsulta oftalmológica y datos de epilepsia mediante la realización de una electroencefalografía.

En los antecedentes personales y familiares, se debe enfocar la búsqueda en crisis epilépticas, migrañas, consumo de fármacos y drogas. $\mathrm{Si}$, tras completar la evaluación del paciente, no queda clara la etiología del proceso, ${ }^{13-15}$ es conveniente pedir una prueba de imágenes para descartar la patología orgánica (tumores, hemorragias, infartos, etc.).

Conocer el síndrome de Alicia en el País de las Maravillas ${ }^{13,14}$ y su relación con distintas patologías, por ejemplo, la migraña, como en el caso presentado, contribuirá a manejar de forma adecuada a estos pacientes y limitar las pruebas complementarias en función de la sospecha clínica. Ante un paciente con sintomatología como la descrita, se debe tener presente su posible relación con una migraña, la cual puede aparecer como manifestación aislada o como aura única no siempre seguida de cefalea. ${ }^{15}$

\section{REFERENCIAS}

1. Cancho Candela R, Hedrera Fernández A. Cefalea en el niño y adolescente. Pediatr Integral. 2015; 19(9):632-9

2. Mortimer J, Kay J, Jaron A. Epidemiology of headache and childhood migraine in an urban general practice using Ad Hoc, Valquist and IHS criteria. Dev Med Child Neurol. 1992; 34(12):1095-101.

3. Durá Travé T, Yoldi Petri ME. Cefaleas agudas recurrentes: características clínicas y epidemiológicas. An Pediatr (Barc). 2005; 62(2):141-6.

4. Gutiérrez-Mata AP, López-Casas J, Ortez-González CI, et al. Características clínicas y evolución de los pacientes con cefalea migrañosa seguidos en la Unidad de Cefaleas de un hospital pediátrico de referencia. Rev Neurol. 2008; 46(6):331-6.

5. Gordon K, Dooley J, Wood E. Prevalence of reported migraine headaches in Canadian adolescents. Can J Neurol Sci. 2004; 31(3):324-7.

6. Headache Classification Committee of the International Headache Society (IHS). The International Classification of Headache Disorders. Cephalalgia. 2013; 33(9):629-808.

7. Corral-Caramés MJ, González-LópezM, López-AbelB, etal. Síndrome de Alicia en el País de las Maravillas como aura persistente de migraña e inicio de enfermedad migrañosa. Rev Neurol. 2009; 48(10):520-2.

8. Asociación Española de Pediatría. Protocolos Diagnósticos y Terapéuticos en Pediátrica. Neurología. 2.da ed. Madrid: AEP; 2008. [Consulta: 2 de noviembre de 2017]. Disponible en: https: / / www.aeped.es/ documentos / protocolosneurologia-en-revision.

9. Shevell MI. Acephalgic migraines of childhood. Pediatr Neurol. 1996; 14(3):211-5.

10. Lippman CW. Certain hallucinations peculiar a migraine. J Nerve Ment Dis. 1952; 116(4):346-51.

11. Todd J. Alice in Wonderland syndrome. Can Med Assoc J. 1955; 73(9):701-4.

12. Losada-Del Pozo R, Cantarín-Extremera V, García Peñas JJ, et al. Características y evolución de los pacientes con síndrome de Alicia en el País de las Maravillas. Rev Neurol. 2011; 53(11):641-8.

13. Liu AM, Liu JG, Liu GW, Liu GT. Alice in Wonderland syndrome: presenting and follow-up characteristics. Pediatr Neurol. 2014; 51(3):317-20.

14. Smith RA, WrightB, BennettS. Hallucinations and illusions in migraine in children and the Alice in Wonderland syndrome. Arch Dis Child. 2015; 100(3):296-8.

15. Ilik F, Ilik K. Alice in Wonderland syndrome as aura of migraine. Neurocase. 2014; 20(4):474-5. 